\title{
Ações de intervenção na formação de professores com base em metodologia tecnológica
}

\author{
Maici Duarte Leite $^{1}$, Francisco Reinaldo ${ }^{1}$, Quelli Cristina da Silva Oliveira ${ }^{2}$ \\ ${ }^{1}$ Depto. Acad. de Informática \\ Universidade Tecnológica Federal do Paraná (UTFPR) \\ Francisco Beltrão - PR - Brasil \\ ${ }^{2}$ Colégio Estadual Mario de Andrade \\ Francisco Beltrão - PR - Brasil
}

\{maicileite, reinaldo\} dutfpr.edu.br, quellidsoliveira@gmail.com

\begin{abstract}
Computer science has transformed society and is no different in the educational space. Thinking about educational methodologies permeated by technology is an aspect that has been receiving investments in physical structures, but it is fragile in the application methodology. In addition to technology, there is application within school spaces. Thus, the research emerges from the perspective of presenting intervention actions in teacher training of students of the Informatics / Computing Degree.
\end{abstract}

Resumo. A informática vem transformando a sociedade e não é diferente dentro do espaço educacional. Pensar em metodologias educacionais permeadas por tecnologia é uma vertente, que vem recebendo investimentos para estruturas físicas, mas está frágil em metodologia de aplicação. Para além da tecnologia tem-se a aplicação dentro dos espaços escolares. Assim, surge a pesquisa na perspectiva de apresentar ações de intervenção na formação de professores, a partir dos alunos do curso de Licenciatura em Informática/Computação.

\section{Introdução}

Dentro dos espaços escolares, a tecnologia vem permeando todas as áreas do conhecimento. Embora a tecnologia seja uma mola propulsora para mudanças significativas em vários aspectos, parece que esta energia acumulada não atinge a mesma velocidade ao contexto educacional. Para a Sociedade Brasileira de Computação (SBC) não se pode almejar uma sociedade tecnologicamente desenvolvida com indivíduos aletrados em pensamento computacional. Conhecimentos básicos nas áreas de Matemática, Filosofia, Física e outras ciências, sugerem que existem alicerces para suportar esta nova sociedade. Sem estratégias a resolver problemas de alta complexidade com a construção de artefatos em linhas de pensamento computacional, não tem-se uma solução breve e replicável [Raabe et al. 2017]. O Model Curriculum for K-12 Computer Science [Tucker 2003] destaca que as profissões do século XXI exigem uma compreensão muito mais ampla que especificamente técnica.

O espaço escolar vem recebendo a demanda de práticas docentes que convergem para o uso da tecnologia. Infelizmente, propostas educacionais carregadas de um contexto ainda não mediado por tecnologia, vem freando a transformação e o impacto que 
VIII Congresso Brasileiro de Informática na Educação (CBIE 2019)

Anais dos Workshops do VIII Congresso Brasileiro de Informática na Educação (WCBIE 2019)

a sociedade almeja. Nesta vertente, tem-se alunos desmotivados, frente a tecnologia que carregam em suas mãos, o smartphone. Outro aspecto a considerar é o uso do laboratório de informática para fins pedagógicos nas escolas, mas o seu modelo organizacional não efetiva o potencial impacto com a tecnologia na aprendizagem. Também não leva em conta as recentes mudanças na sociedade trazidas pela ubiquidade das tecnologias móveis e da internet [CIEB 2019].

De uma maneira geral, as políticas públicas incentivam uma educação de qualidade com os investimentos aos espaços escolares. Contudo, o paradigma não tem se transformado na mesma velocidade dentro das escolas públicas. Enquanto as escolas públicas se estruturam com os laboratórios de informática com computadores de mesa, as escolas privadas avançam em investimentos com outras novas tecnologias portáteis e vestíveis. Vale destacar que as escolas privadas foram as primeiras a instalarem laboratórios de informática com computadores de mesa como um novo potencial para alavancar a aprendizagem, e foram as primeiras a encerrar as atividades neste contexto [Tribeck et al. 2017]. Portanto, as novas relações de aprendizagem por meio da tecnologia ganham espaço expressivo e a realidade presente em escolas públicas traz uma reflexão pertinente, a viabilidade e o uso do laboratório de informática na perspectiva educacional aplicada.

Em uma breve reflexão do panorama das possíveis habilidades de um profissional, torna-se relevante esclarecer que o contexto educacional das escolas públicas não está dinâmico. Ignora-se a utilização dos dispositivos móveis em M-learning [Saccol et al. 2010] como ferramenta para auxiliar o professor a promover no aluno sua aprendizagem, autonomia, criticidade e criatividade [Reinaldo et al. 2016a]. Também ofusca o papel do professor como personagem de mediador da interação entre a tríade aluno, conhecimento e computador. O que se observa na prática escolar é a fragilidade da formação do professor para o exercício deste papel [Reinaldo et al. 2016b]. Também a escola não demanda de dispositivos móveis a contemplar um por aluno ou mesmo contemplam metodologias de ensino diferenciadas na prática docente, ou ainda, tenham uma proposta metodológica baseada neste panorama.

O estudo de [Leite et al. 2017] identificou as demandas das escolas municipais de Francisco Beltrão - Paraná, nos aspectos que envolvem o uso da tecnologia como meio de transmissão e aquisição de conhecimento, seja por parte do aprendiz ou do professor. Os resultados demostraram que a reconstrução dos processos de ensino e aprendizagem é necessária no exercício docente relacionado à Computação nas escolas municipais, assim como: (i) mais investimentos na formação de professores para uso de recursos tecnológicos, (ii) aplicação do Pensamento Computacional, (iii) mais habilidade que metodologia. Nesta vertente, com a evolução e disseminação de novas metodologias com ênfase em tecnologias educacionais percebeu-se a necessidade de novamente levantar dados atuais a fim de validar/planejar ações para formação de professores, dentro dos espaços escolares. Nesta vertente, o objetivo deste artigo foi apresentar ações de intervenção na formação de professores com base em metodologia tecnológica - para um contexto que emerge rapidamente e não somente no espaço educacional.

\section{Metodologia}

A primeira etapa deste artigo foi desenvolvida no segundo semestre de 2017, que resultou no trabalho [Leite et al. 2017], para compreender se a aplicação da proposta do Pensa- 
VIII Congresso Brasileiro de Informática na Educação (CBIE 2019)

Anais dos Workshops do VIII Congresso Brasileiro de Informática na Educação (WCBIE 2019)

mento Computacional nas escolas estaduais estava limitada à tecnologia ou ao currículo dos professores. A resposta obtida foi que a limitação estava no currículo dos professores no uso de tecnologias no espaço educacional. Assim, desenvolveu-se uma segunda etapa de pesquisa, que ocorreu no segundo semestre de 2019, para investigar ações de intervenção na formação de professores com base em metodologia tecnológica.

O levantamento de dados aconteceu entre os anos de janeiro de 2018 e julho de 2019, por consulta em 17 bancos de dados públicos das escolas estaduais pertencentes ao núcleo regional de educação do estado do Paraná. Os bancos de dados estão disponibilizados pelos subsistemas da CELEPAR Paraná, tais como PRD Estatística ${ }^{1}$ e $\mathrm{SDI}^{2}$. As consultas públicas alimentaram uma base de dados de pesquisa definida neste artigo então desmembrada em quatro dimensões, conforme [Leite et al. 2017], sendo (i) Infraestrutura e Equipamentos Informáticos, (ii) Acesso à internet nas Salas de Aulas, (iii) Ferramentas e Programas mais utilizados nas Salas de Informática (iv) internet vs. Atividades Escolares.

Obedecendo aos critérios de filtragem amostral, tem-se que das 16 escolas públicas estaduais da região [Leite et al. 2017], somente 12 foram selecionadas para estudo. Assim, tem-se a educação básica contemplada no Ensino Fundamental e Médio com Ensino Técnico integrado. As 12 escolas participantes incluídas são compostas por alunos em sua maioria pertencentes às classes socioeconômicas B e C [Yaccoub 2011], com acesso a computadores na residência, na escola ou ambas.

\section{Resultados}

\subsection{Descritor das Dimensões}

Dimensão I - Infraestrutura e equipamentos de informática: $O$ modelo atual de laboratório de informática, presente em grande parte das escolas brasileiras, consiste em dispor o maior número possível de computadores de mesa, lado a lado, em uma sala [CIEB 2019]. Embora pareçam remodelar às aulas de datilografia do início do século XX e, ainda que possa funcionar no objetivo de capacitar alunos no uso instrumental de aplicações básicas de informática, não é efetivo para impactar profunda e positivamente o ensino e a aprendizagem [Trucano 2011].

A Tabela 1 apresenta que existe estrutura compatível, embora o uso da metodologia tecnológica seja um item de discussão complexo.

Tabela 1. Infraestrutura vs Equipamentos

\begin{tabular}{lcc}
\hline & Primeira Etapa(\%) & Segunda Etapa(\%) \\
\hline Razoável infraestrutura & 30 & 36 \\
Boa infraestrutura & 30 & 28 \\
Computadores e sala sem condições & 35 & 33 \\
Não possui sala de computadores & 5 & 3 \\
\hline
\end{tabular}

Fonte: O autor.

\footnotetext{
${ }^{1}$ Análise de Uso de Computadores nas Escolas por www.prdestatistica.seed.pr.gov.br

${ }^{2}$ Sistema de Diagnóstico Instantâneo por www.sdi . seed.pr.gov .br/
} 
VIII Congresso Brasileiro de Informática na Educação (CBIE 2019)

Anais dos Workshops do VIII Congresso Brasileiro de Informática na Educação (WCBIE 2019)

No que se refere a segunda etapa, é possível observar na Tabela 1 que $64 \%$ da infraestrutura dos laboratórios de informática está entre razoável e boa, enquanto 33\% apresenta infraestrutura antiga, mas investimentos do governo estadual permitem alterar este cenário.

Assim, a disponibilidade da infraestrutura e equipamentos é relevante, mas o uso é uma outra realidade e parece que a inserção da tecnologia no espaço escolar está consolidada em torno dos laboratórios de informática. Nesse formato, [CIEB 2019] coloca que o foco é centralizar e monopolizar os equipamentos voltados para o ensino e a aprendizagem, enquanto que o futuro aponta para a integração da tecnologia a todos os ambientes das escolas e ao dia a dia do ensino.

Dimensão II - Acesso à internet nas salas de aulas: Em relação ao acesso à internet nas salas de aulas, a Tabela 2 apresenta um resultado que favorece o acesso à internet.

Tabela 2. Acesso à internet das salas de informática

\begin{tabular}{lcc}
\hline & Primeira Etapa (\%) & Segunda Etapa (\%) \\
\hline É precária & 15,8 & 12,3 \\
Não tem acesso a internet & 52,6 & 20,5 \\
Funciona, mas instável & 15,8 & 25,0 \\
Estável e inadequado & 10,5 & 12,2 \\
Estável e adequado & 5,3 & 30,0 \\
\hline
\end{tabular}

Fonte: $\mathrm{O}$ autor.

Um estudo do Centro Regional de Estudos para o Desenvolvimento da Sociedade da Informação ${ }^{3}$ informou que o acesso à internet sem fio cresce nas escolas públicas, mas a velocidade é um desafio. Enquanto o uso da rede wifi subiu de $84 \% \mathrm{em}$ 2015 para $91 \%$ em 2016, a conexão de $45 \%$ das escolas ainda não ultrapassa a velocidade de 4 Mbps [NIC 2017]. Embora o Paraná tenha o Programa Escola Conectada [CASA CIVIL DO GOVERNO DO ESTADO DO PARANÁ 2018], as ações de uso ainda contemplam a pesquisa por motores de busca. Assim, a Tabela 2 mostrou que $67 \%$ das escolas tem acesso a internet, embora dados revelaram que mais de $20 \%$ das escolas ainda não possuem acesso à internet, sendo passível de investimento pela associação de pais e mestres.

Dimensão III - Ferramentas e programas mais utilizados nas salas de informática: Em relação às Ferramentas e Programas mais utilizados nas Salas de Informática, a Tabela 3 revelou que as escolas não possuem acesso constante à internet.

De acordo com a Tabela 3, mais de 31\% apresentam softwares utilitários. Esta informação é importante pois pode ser ofertada formação para professores com base na metodologia tecnológica. Também, é possível desenvolver ações relevantes, sinalizadas no item internet e softwares diversos.

\footnotetext{
${ }^{3}$ www.cetic.br
} 
VIII Congresso Brasileiro de Informática na Educação (CBIE 2019)

Anais dos Workshops do VIII Congresso Brasileiro de Informática na Educação (WCBIE 2019)

Tabela 3. Ferramentas e programas utilizados nas salas de informática

\begin{tabular}{lcc}
\hline & Primeira Etapa (\%) & Segunda Etapa (\%) \\
\hline Internet e softwares diversos & 17,1 & 23,0 \\
Não há acesso a internet & 25,7 & 15,3 \\
Não sei/sem resposta & 8,6 & 5,4 \\
Softwares utilitários & 17,1 & 31,1 \\
Softwares educativos diversos & 25,7 & 18,0 \\
Software livre & 5,7 & 7,2 \\
\hline
\end{tabular}

Fonte: O autor.

Neste sentido, já é possível perceber um avanço em algumas iniciativas em evidência nas escolas que possuem cultura tecnológica mais amadurecida, com aulas de robótica educacional, clubes de programação, criação de jogos digitais, simuladores e atividades interdisciplinares com "computação desplugada" [Bell et al. 2011]. Novamente, é uma iniciativa presente em escolas privadas, embora com iniciativas na escola pública, através de programas como Programa de Iniciação à Docência - PIBID e estágios, desenvolvidos por alunos da Licenciatura em Informática/Computação. Vale destacar é uma ação positiva, uma vez que intensifica o diálogo entre as discussões sobre uso de tecnologias nos processos de ensino.

Dimensão VI - internet vs. atividades escolares: Os dados presentes na Tabela 4 revelaram que os softwares educativos apresentam fragilidades no viés de aplicação dentro do espaço escolar. Infere-se que, infelizmente, o uso das salas de informática está somente pela manipulação de objetos, sem um motivo ou aplicação direta em seu cotidiano escolar.

Tabela 4. Fatores relacionados ao desinteresse dos alunos quanto às atividades na sala de informática

\begin{tabular}{lcc}
\hline & Primeira Etapa (\%) & Segunda Etapa (\%) \\
\hline Softwares desatualizados & 11,5 & 13,0 \\
Poucos computadores & 26,9 & 30,2 \\
Não tem acesso à internet & 30,8 & 36,0 \\
Sem familiaridade com softwares & 11,5 & 10,8 \\
Não sei/sem resposta & 7,7 & 3,0 \\
Outro & 11,5 & 7,0 \\
\hline
\end{tabular}

Fonte: O autor.

Especificamente, os resultados encontrados na Tabela 4 evidenciam que pouco acesso a internet é relevante, dada a dinamicidade da tecnologia, hoje é quase que impossível pensar no uso de um computador sem acesso à internet, e muito menos aplicar ao contexto educacional, conforme se evidencia nos $36 \%$. Embora, em relação a quantidade de computadores com aproximadamente $30 \%$, ser um fator considerável, pode-se dizer que muitos alunos, senão a maioria podem utilizar o smartphone, sendo um problema de menor valor, quem sabe o uso adequado e aplicação seja o fator mais relevante para 
VIII Congresso Brasileiro de Informática na Educação (CBIE 2019)

Anais dos Workshops do VIII Congresso Brasileiro de Informática na Educação (WCBIE 2019)

dinamizar o interesse.

\subsection{Metodologia Tecnológica}

A inserção de novas propostas metodológicas nos espaços escolares vem da demanda crescente da inclusão das novas tecnologias nos mais diferentes contextos sociais [Oliveira et al. 2017]. Assim o curso de Licenciatura em Informática ou Computação visa atender a demanda de formação de professores capazes de explorarem os recursos tecnológicos e desenvolvimento de tecnologias para a educação, bem como ensinar a Computação na escola. No caso deste estudo, a disciplina de Prática em Computação se dispôs a analisar os resultados e propor atividades na perspectiva do estudo. Para atender a proposição pensou-se em três desafios no viés de formação de professores da educação pública [Leite et al. 2017]: (i) laboratórios de informática, (ii) tecnologias aplicadas e (iii) Pensamento Computacional. Por se entender que, somente laboratórios de informática sem acesso a internet merecem uma atenção considerável, dada a complexidade no uso em relação aos softwares instalados, as tecnologias aplicadas tendem a contribuir dentro das áreas específicas de cada conteúdo curricular e o Pensamento Computacional é um paradigma que vem atendendo as demandas na área.

\subsection{Laboratórios de informática}

O programa Escola Conectada é um programa do Estado do Paraná e tem como objetivo modernizar os sistemas de informática dos colégios da rede estadual. Os investimentos giram em torno de R $\$ 283$ milhões para aquisição de equipamentos e melhorias da rede de internet das escolas. É o maior pacote de investimentos da história da educação do Paraná, ao todo, foram destinados aproximadamente $\mathrm{R} \$ 600$ milhões para projetos de infraestrutura de escolas e Apaes [SECRETARIA DE EDUCAÇÃO DO PARANÁ 2018].

O Paraná Digital foi lançado em 2003 em uma parceria das secretarias da Educação, da Ciência e Tecnologia, da Companhia de Informática do Paraná (Celepar), da Companhia Paranaense de Energia Elétrica (Copel) e da Universidade Federal do Paraná (UFPR). O programa Lançado em 2003 tem como objetivo levar a tecnologia da internet a todas as escolas estaduais através da fibra ótica da Copel, além de conjugar as estruturas dos laboratórios de informática, das TVs multimídia e do Portal Dia a Dia Educação. Mesmo com o aporte de uma universidade pública (UFPR) para desenvolver a pesquisa e softwares e hardwares, no que se refere a prática docente e/ou formação de professores e aplicação no espaço escolar, as fragilidades sobressaem.

A implantação de recursos tecnológicos causou impacto no ambiente escolar, sinalizou a demanda de revisão de formação e prática docente, mas não ocasionou muitas mudanças. O cotidiano escolar parece estar em outra velocidade. O professor que demonstrava no quadro com giz, agora conta com novos recursos digitais que permitem projetar ou obter imagens de alta qualidade através da TV Multimídia. Porém um estudo de [Basniak 2016] destaca que os recursos não foram suficientes para modificar a prática do professor no sentido de uso para potencializar a aprendizagem e, também nos cursos para a formação do professor, os novos recursos também não foram discutidos nesse sentido.

Embora não se tenha em números estas contribuições na educação anteriormente apresentadas, novos equipamentos para alavancar a infraestrutura estão chegando às escolas. Então percebe-se um movimento crescente, mas evidencia-se a necessidade de 
VIII Congresso Brasileiro de Informática na Educação (CBIE 2019)

Anais dos Workshops do VIII Congresso Brasileiro de Informática na Educação (WCBIE 2019)

formações dos professores ligadas às tecnologias educacionais integradas ao espaço escolar e ao trabalho do professor.

Nesta vertente, para os laboratórios de informática faz-se a seguinte proposição de instalação de softwares livres para uso didático dos alunos, tais como GeoGebra específico para o ensino de matemática e física - planilha eletrônica, ensino baseado em projetos (laboratório como suporte para resolução de problemas), softwares de simulação, produção de vídeos, edição de imagens, preparação de Podcast (arquivo digital de áudio transmitido através da internet).

Como pode-se perceber o uso do laboratório de informática, sem acesso à internet é limitado e demanda de expertise dos professores em computação, além das suas áreas de ensino. Neste momento entram os egressos da Licenciatura em Informática/Computação, com a responsabilidade pela formação de professores para o exercício da docência em Computação na Educação e para atuar no projeto, no desenvolvimento, na avaliação e na gestão de sistemas educacionais e de tecnologias contemporâneas, relacionadas à Computação articulada à Educação [Raabe et al. 2017].

\subsection{Tecnologias aplicadas}

As Diretrizes Curriculares Nacionais para os cursos de graduação em Computação, na Resolução CNE/CES n ${ }^{\circ}$ 05/2016 [MEC 2016] destacam que os cursos de Licenciatura em Computação têm como objetivo principal preparar professores para formar cidadãos com competências e habilidades necessárias para conviver e, prosperar em um mundo cada vez mais tecnológico e global e que contribuam para promover o desenvolvimento econômico e social de nosso País. Ou seja, o sistema computacional com funcionalidade pedagógica ou que necessita de assistência para seu uso, requer a participação dos Licenciados em Computação.

De forma muito objetiva pode-se dizer que enquanto os recursos tecnológicos são representados por equipamentos, aplicativos e softwares, a metodologia diz muito sobre o formato de como será explorado, que remete ao planejamento do professor no contexto de uso. Assim, percebe-se que existe fragilidade no que se refere a metodologia tecnológica e a grade curricular, dada a diversidade de software e aplicativos, além de plataformas de ensino on-line. Nesta via, o egresso da Licenciatura em Informática/Computação pode desenvolver atividades para a formação do professor regente da disciplina que irá delinear a estratégia de ensino e uso das ferramentas tecnológicas. Contudo, demandará conhecimento específico da área do conhecimento, ainda que a experiência do profissional seja ampla e consistente.

Na perspectiva da metodologia tecnológica o uso com aplicação por área de componente curricular é vasto, mas vale destacar que o acesso à internet impulsiona as ações de tomada de decisão. A metodologia tecnológica faz a conexão, uma vez que os meios digitais representam uma infinidade de possibilidades que precisam ser filtradas e adaptadas às realidades de ensino. Então identificar a potencialidade de aplicação dos recursos na prática pedagógica é tarefa do professor da disciplina. É fundamental que o professor crie e experimente práticas pedagógicas inovadoras, entendidas como aquelas especificamente contrapostas a perspectivas instrucionais e instrumentais de ensino [CIEB 2019]. Outra perspectiva de uso da informática aplicada que vem surgindo são os aplicativos e as plataformas que podem ser utilizadas dentro da escola. Como ponto positivo, vem incluso 
VIII Congresso Brasileiro de Informática na Educação (CBIE 2019)

Anais dos Workshops do VIII Congresso Brasileiro de Informática na Educação (WCBIE 2019)

com o próprio material didático e pode se dizer de interatividade que permitem aos alunos explorar elementos visuais, sonoros e até sinestésicos - a partir do conteúdo apresentado, o professor é o tutor.

\subsection{Pensamento Computacional}

A tecnologia é indispensável para a resolução de problemas, dos mais simples aos mais complexos do cotidiano, e o Pensamento Computacional (PC) surge neste viés com consenso entre os educadores, como demanda de desenvolvimento de habilidades de informática. Então para uma geração surge em contato com tecnologia, a demanda vai além do conhecimento de ferramentas básicas e de noções como comunicação na internet.

O Model Curriculum for K-12 Computer Science aconselha o desenvolvimento de habilidades computacionais na educação básica, no sentido de promover múltiplos caminhos profissionais, desenvolver a capacidade de resolver problemas, apoiar e relacionar-se com outras ciências e motivar os estudantes [Tucker 2003].

O Pensamento Computacional (PC) incorpora inovações tecnológicas no meio pedagógico e promove o dinamismo nas técnicas e métodos tradicionais. O conceito proposto por [Wing 2006], se refere à capacidade de sistematizar, representar, analisar e resolver problemas. O autor ainda caracteriza o PC com os seguintes elementos: (i) conceptualização (organização dos conceitos) e não programação; (ii) habilidade fundamental, não mecânica; (iii) forma que humanos, não computadores, pensam; (iv) pensamento complemento e que combina pensamento matemático e de engenharia; (v) composto por ideias, não somente software e hardware; (vi) para todas as pessoas, em todos os lugares.

Assim deve pensar em aulas que desenvolvam habilidades que incluem identificação de padrões e sequências, criação de algoritmos, formulação de testes para encontrar e solucionar erros, redução do geral para o específico e expansão do específico para o geral. No âmbito de estimular a capacidade para resolver problemas, decompondo-os e os solucionando, através de planejamento, para execução. Portanto, propor atividades que estimulem os alunos escreverem o passo a passo para a resolução de um problema que poderiam ser replicados.

Confirma-se a relevância de uma capacidade criativa, crítica e estratégica de usar os fundamentos do PC nas mais diversas áreas de conhecimento com a finalidade de resolver problemas de uma maneira individual ou colaborativa. O PC é uma alternativa para mostrar aos estudantes o outro lado das ferramentas que fazem parte do seu cotidiano e que dão suporte na resolução de problemas.

\section{Conclusões}

Os laboratórios de informática ainda são os espaços que concentram a maior parte dos computadores para fins pedagógicos, dentro das escolas públicas [CIEB 2019], embora o uso da tecnologia nos espaços escolares não é realidade em grande parte das escolas, quer seja por uma questão de infraestrutura ou pela falta de formação adequada para o corpo docente, ainda é bem distante da ideal. E se acentuam com as medidas adotadas pelo poder público descontextualizadas da realidade e as demandas da comunidade educacional.

O presente paradigma educacional demanda de uma reflexão do impacto da tecnologia na aprendizagem, mas não leva em conta as recentes mudanças nos perfis profissionais trazidas pela ubiquidade das tecnologias móveis e da internet, como princípio inicial. 
VIII Congresso Brasileiro de Informática na Educação (CBIE 2019)

Anais dos Workshops do VIII Congresso Brasileiro de Informática na Educação (WCBIE 2019)

E muito menos da infraestrutura disponível, parece que a solução não está nos laboratórios de informática, nem no acesso à internet, mas sim na formação de professores para tecnologias contextualizadas com seu conteúdo curricular e realidade do espaço escolar, embora esteja claro que o professor pode transitar em mais de um espaço. Embora seja uma utopia, vale a reflexão.

Então retoma-se que os investimentos para a ampliação da presença dos laboratórios nas escolas públicas é fato, a parcela de escolas públicas que ainda não possui laboratórios de informática deve ser muito pequena e já deve estar na lista de escolas que conseguirão a infraestrutura, mas é urgente a reflexão sobre a necessidade da comunidade escolar integrar tecnologias à educação e capacitar seus docentes para uso prático, eficiente e pedagógico dessas tecnologias.

Assim começa a surgir a difusão desses espaços de inovação nas escolas das redes públicas de ensino, aliada as formações ativas e continuadas para os professores no uso pedagógico da tecnologia, abrindo caminho para um ciclo de inovação pedagógica que tem potencial para alavancar o ensino básico público de forma transformadora.

\section{Referências}

Basniak, M. I. (2016). Políticas de tecnologias na educação: o programa paraná digital. Educar em Revista, pages 305 - 319.

Bell, T., Witten, L., and Fellows, M. (2011). Computer Science Unplugged: Ensinando Ciência da Computação sem o uso do computador.

CASA CIVIL DO GOVERNO DO ESTADO DO PARANÁ (2018). Decreto 8801 - 19 de fevereiro de 2018. Publicado no Diário Oficial n. 10132 de 20 de Fevereiro de 2018.

CIEB (2019). Criação de espaços de inovação nas escolas: repensando o laboratório de informática. Technical report, Centro de Inovação para a Educação Brasileira.

Leite, M., Reinaldo, F., Maschio, E., Marczal, D., and Oliveira, C. (2017). Pensamento computacional nas escolas: Limitado pela tecnologia, infraestrutura ou prática docente? Anais dos Workshops do Congresso Brasileiro de Informática na Educação, 6(1): 1002 .

MEC (2016). Resolução 5, de 16 de novembro de 2016. Publicado online.

NIC, N. (2017). Pesquisa sobre o uso das tecnologias de informação e comunicação nas escolas brasileiras. Technical Report 1, CETI.BR, CGI.BR.

Oliveira, W., Hinterholz, L., and Silva, C. (2017). Licenciatura em computação: Desafios e oportunidades na perspectiva do professor. page 705 .

Raabe, A. L. A., Zorzo, A. F., Frango, I., Ribeiro, L., Granville, L. Z., Salgado, L., da Cruz, M. J. K., Bigolin, N., Cavalheiro, S. A. C., and Fortes, S. (2017). Referenciais de formação em computação: Educação básica. Technical report, Sociedade Brasileira de Computação. https://www.sbc.org.br/documentos-da-sbc/send/131-curriculos-dereferencia/1166-referenciais-de-formacao-em-computacao-educacao-basica-julho2017.

Reinaldo, F., Magalhães, D. R., Reis, L. P., Gaffuri, S., Freddo, A., and Hallal, R. (2016a). Impasse aos desafios do uso de smartphones em sala de aula: Investigação por grupos focais. RISTI-Revista Ibérica de Sistemas e Tecnologias de Informação, (19):77-92. 
VIII Congresso Brasileiro de Informática na Educação (CBIE 2019)

Anais dos Workshops do VIII Congresso Brasileiro de Informática na Educação (WCBIE 2019)

Reinaldo, F., Renó, D., Reis, L., Gaffuri, S., Freddo, A., and Hallal, R. (2016b). Uso de smartphones na educação: Avaliação por grupos focais. In Atas - Investigação Qualitativa em Educação, pages 769-778.

Saccol, A. Z., Reinhard, N., Schlemmer, E., and Barbosa, J. L. V. (2010). M-learning (mobile learning) in practice: a training experience with it professionals. JISTEM Journal of Information Systems and Technology Management, 7:261 - 280.

SECRETARIA DE EDUCAÇÃO DO PARANÁ (2018). Programa amplia estrutura de informática na rede estadual. http://www.educacao.pr.gov.br/modules/noticias/article.php?storyid=7451\&tit=Programaamplia-estrutura-de-informatica-da-rede-estadual.

Tribeck, P. M. A., Reinado, F., and Leite, M. D. (2017). Edutracesys como objeto de aprendizagem na remediação de erros. Espacios.

Trucano, M. (2011). School computer labs: A bad idea? https://blogs.worldbank.org/edutech/computer-labs. EduTech.

Tucker, A. (2003). A model curriculum for k-12 computer science: Final report of the acm k-12 task force curriculum committee. Technical report, New York, NY, USA. ACM Order No.: 104043.

Wing, J. M. (2006). Computational thinking. Commun. ACM, 49(3):33-35.

Yaccoub, H. (2011). A chamada "nova classe média": cultura material, inclusão e distinção social. Horiz. antropol., 17:197 - 231. 